\title{
Incidence of early complications in the posterolateral approach to posterior malleolus fractures
}

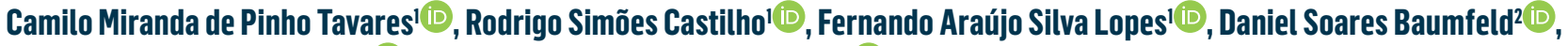 \\ Thiago Alexandre Alves Silva ${ }^{3}$, Roberto Zambelli de Almeida Pinto ${ }^{1}[$ \\ 1. Hospital Mater Dei, Belo Horizonte, MG, Brazil. \\ 2. Hospital Felício Rocho, Belo Horizonte, MG, Brazil. \\ 3. Hospital Madre Teresa, Belo Horizonte, MG, Brazil.
}

\begin{abstract}
Objective: To ascertain the incidence of early complications in the posterolateral approach to open reduction and internal fixation of posterior malleolar fractures and identify possible risk factors related to occurrence of these complications.

Methods: Retrospective study carried out in three tertiary hospitals. Patients who underwent open reduction and internal fixation of posterior malleolus fractures via the posterolateral route were divided into two groups: with versus without delayed postoperative healing. To assess risk and protective factors for the outcome of delayed healing, we evaluated the time between trauma and surgery, whether the patient had a fracture-dislocation of the ankle, and whether external fixation was performed before surgery. We also evaluated whether improvement in operative technique led to a reduction in complication rates.
\end{abstract}

Results: A total of 43 individuals who underwent surgical correction of posterior malleolus fractures via the posterolateral route between 2013 and 2018 were included. Of these, 19 (44.2\%) had skin complications that led to delayed healing. Skin complications occurred more frequently at the beginning of the learning curve of the surgeons involved; the incidence up to the year 2016 was $56.3 \%$, decreasing significantly to $37 \%$ from 2017 onward.

Conclusion: Patients who undergo open reduction and internal fixation of posterior malleolus fractures via the posterolateral route at the beginning of the learning curve are a greater risk of developing skin complications, demonstrating the importance of this approach being restricted to more experienced surgeons.

Level of Evidence III; Prognostic Studies; Retrospective Study.

Keywords: Ankle; Internal fixation; Surgical wound; Tibial fractures.

\section{Introduction}

Malleolar fractures are extremely common injuries in the foot and ankle surgeon's routine practice. Indeed, they are the fourth most common indication for orthopedic surgery, with an incidence of 187 per 100,000 person-years ${ }^{(1)}$. Isolated lateral malleolus fracture accounts for $66 \%$ of ankle fractures; $25 \%$ are bimalleolar, and only $7 \%$ are trimalleolar ${ }^{(2-4)}$.

Posterior malleolus fracture (PMF) carries a worse prognosis, since it is associated with higher odds of subsequent osteoarthrosis ${ }^{(5,6)}$ (Figures $1 A$ to $D$ ). Internal fixation of the posterior malleolus remains a controversial procedure. Tradi- tionally, it is indicated when the fragment contains more than $25 \%$ of the articular surface, as measured on lateral radiography, and is displaced more than $2 \mathrm{~mm}^{(7)}$. In the last decade, research attention has increasingly focused on the posterior malleolus fragment itself, as involvement of the fibular notch of the distal tibia and the presence of an interposed articular fragment were understood to be of greater therapeutic relevance than fragment size and the extent of the fractured joint surface area(5).

Simple, undisplaced PMFs can be fixed percutaneously with minimally invasive techniques( ${ }^{(8)}$. However, this technique has
Study performed at the Hospital Mater Dei, Hospital Felicio Rocho and Hospital Madre Teresa, Belo Horizonte, MG, Brazil.

Correspondence: Camilo Miranda de Pinho Tavares. 310/601 Otoni St., Belo Horizonte, MG, Brazil, Zip Code: 30150-270. E-mail: camiloptavares@gmail.com Conflicts of interest: none. Source of funding: own. Date received: July 31, 2020. Date accepted: August 03, 2020. Online: August 30, 2020.
How to cite this article: Tavares CMP; Castilho RS; Lopes FAZ; Baumfeld DS; Silva TAA; Pinto RZA. Incidence of early complications in the posterolateral approach to posterior malleolus fractures. J Foot Ankle. 2020;14(2):178-82. 
some limitations, such as indirect anatomical reduction. For comminuted fractures or those with interposed fragments, open reduction and internal fixation (ORIF) is recommended ${ }^{(9)}$.

The posterolateral approach (PLA) provides an alternative for effective ORIF of any type of posterior malleolar fractu$r \mathrm{e}^{(10)}$. This approach also provides access to the distal fibula, where a plate can be placed posteriorly ${ }^{(11-13)}$. Despite the advantages of the PLA, early complications can affect healing for still-unknown reasons ${ }^{(14)}$.

Within this context, the present study was designed to ascertain the incidence of early complications in the posterolateral approach to ORIF of posterior malleolar fractures and identify possible risk factors related to occurrence of these complications.

\section{Methods}

This study was approved by the relevant Institutional Review Board and registered on the Plataforma Brasil database under CAAE (Ethics Evaluation Submission Certificate) number 28763919.9.0000.5128.

This is a retrospective study to determine the occurrence of complications after open reduction and internal fixation of posterior malleolus fractures via the posterolateral approach. All consecutive patients with fracture or fracture-dislocation of the ankle who underwent ORIF via the posterolateral route for a PMF with displacement greater than $2 \mathrm{~mm}$ or interposition of joint fragments, between the years 2013 and 2018, at three tertiary hospitals were included in the study.

Patients were divided into two groups: those who had postoperative skin complications and those who did not. For the purposes of this study, skin complications were defined broadly, from wound dehiscence treated conservatively to superficial and deep infections requiring more aggressive treatment, and healing was considered delayed if it took longer than 3 weeks. The exclusion criterion was failure to perform PMF correction via the posterolateral approach.

To assess for risk and protective factors for the outcome (skin complications), we evaluated the time between trauma and surgery, whether the patient had a fracture-dislocation of the ankle, and, finally, whether external fixation was performed for damage control before surgery.

For statistical analysis of whether any factors contributed to the presence of skin complications, a univariate analysis was initially carried out via logistic regression, using the stepwise method $^{(15)}$ to select the variables of interest. For multivariate analysis, the backward method(15) was applied. A significance level of $5 \%$ was adopted, and variables with a $p$-value slightly above that value were also accepted, although considered only marginally significant. To assess the goodness of fit and predictive ability of the logistic regression model, the Hosmer-Lemeshow test ${ }^{(16)}$, Nagelkerke's pseudo- $R^{2(17)}$, and the AUC (area under the ROC curve) were used. All statistical analyses were carried out in $\mathrm{R}$ version 3.6.3. To ascertain whether operative technique improved while the surgeon was learning and once the surgeon had acquired experience with the posterolateral approach and subsequent technique, Fisher's exact test was performed with the years 2013 to 2018 and occurrence skin complications as the variables.

\section{Surgical technique}

The patient is placed in the prone position. A posterolateral incision is made longitudinally on the distal aspect of the leg, midway between the Achilles tendon and the fibula (Figure 1E). The sural nerve and small saphenous vein are identified and protected, since the nerve runs from central to lateral to the Achilles tendon, approximately $10 \mathrm{~cm}$ from the attachment of the latter at the calcaneus ${ }^{(18)}$. The posterior fascia of the leg is incised down to the flexor hallucis longus muscle and tendon. This structure is reflected laterally, simultaneously providing access to the posterior malleolus and protecting the midline neurovascular structures.

Fixation of the posterior malleolus can be performed with a posterior antishear plate or with traction screws placed in the posterior to anterior orientation, depending on the need for stabilization and fragment size. The posterior malleolus must be fixed before any other lesions, in order to allow better manipulation and fluoroscopic visualization of the reduction without other hardware getting in the way (Figures $1 \mathrm{~F}$ to $\mathrm{J}$ ).

The same route is habitually used at our service for identification and fixation of the fibular fracture, when present. The proximal fascia of the fibular tendons is incised and opened longitudinally, and the tendons may be reflected medially or laterally. Fixation is performed posterior to anterior, which, in certain fracture patterns, can provide greater biomechanical stability and an antishear stop ${ }^{(19)}$

\section{Results}

A total of 43 individuals were included, 28 of whom were female, with a mean age of 41.5 years (range, 25-65). All had PMF. Delayed healing occurred in 19 patients (44.2\%).

External fixation for damage control was performed prior to definitive surgical treatment in 31 patients (72.1\%). Twenty-three patients $(53.4 \%)$ presented with a fracture dislocation. The mean time elapsed between trauma and definitive treatment was 7.6 days (range, 0-30; standard deviation [SD], 6.2). The mean time from surgery to complete healing of the operative wound was 4.7 weeks (range, 2-17; SD, 4.4) (Tables 1 and 2).

The incidence of skin complications was not significantly associated with time between trauma and surgery $(p=0.766)$, fracture-dislocation of the ankle $(p=0.920)$, or use of external fixation before definitive repair $(p=0.376)$ (Table 3 ).

Skin complications occurred more frequently at the beginning of the learning curve of the surgeons involved; the incidence up to the year 2016 was $56.3 \%$, decreasing significantly to $37 \%$ from 2017 onward (Figure 2). No patient needed major intervention for wound closure.

Sample size calculation showed that, for a significance level of $5 \%$, a moderate effect size, and a statistical power of $80 \%$, each group required a minimum of 67 patients. 

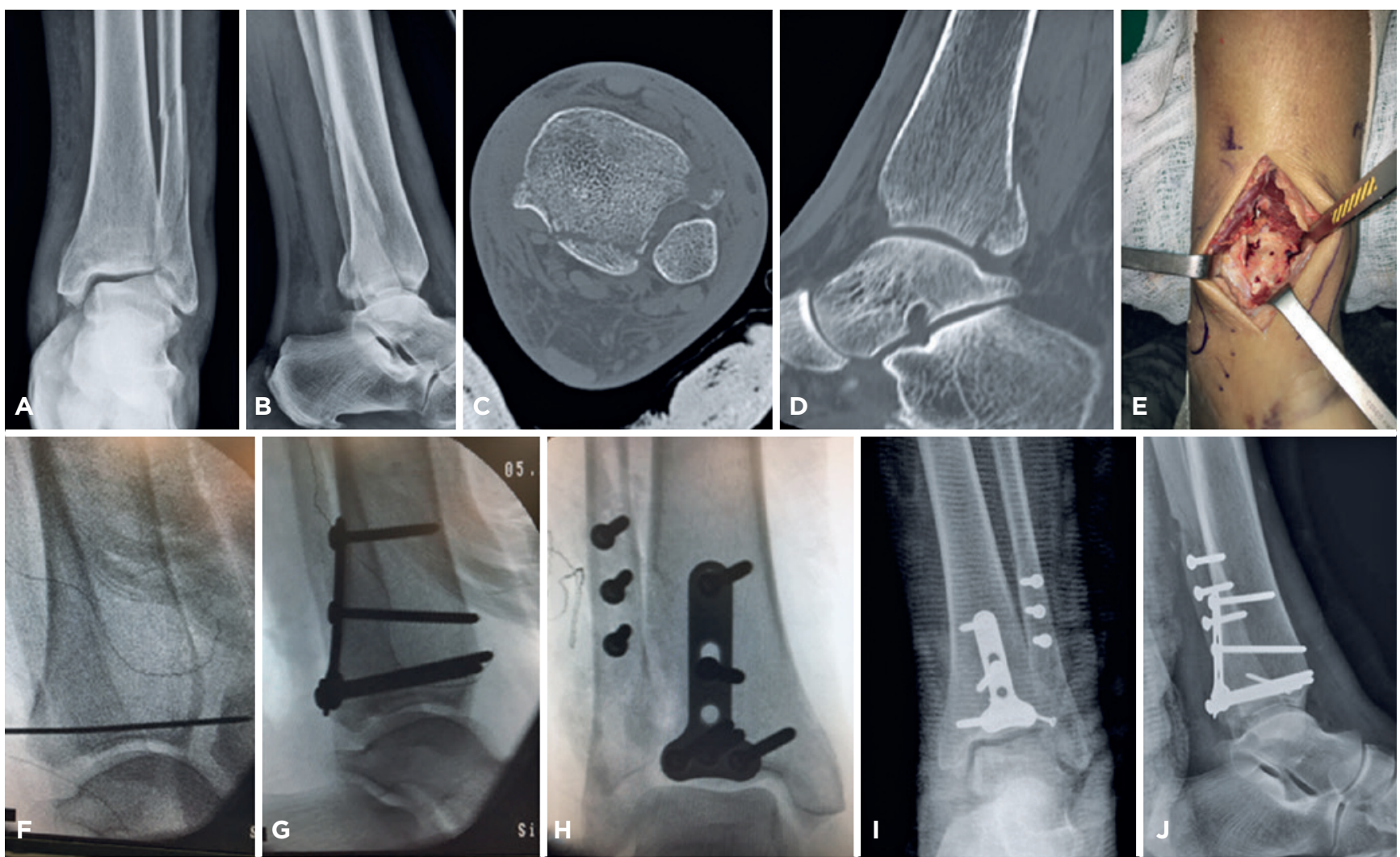

Figure 1. Posterior malleolus fracture (A to D). Posterolateral access with structures reflected for visualization of the posterior malleolus (E); internal fixation of the posterior malleolus and fibula through the posterolateral approach (F to J).

Table 1. Descriptive analysis of qualitative variables

\begin{tabular}{lccc}
\hline \multicolumn{1}{c}{ Variable } & & N & $\%$ \\
\hline Skin lesion & No & 24 & $55.8 \%$ \\
Posterior malleolus fracture & Yes & 19 & $44.2 \%$ \\
& No & 0 & $0 \%$ \\
Fracture-dislocation & Yes & 43 & $100 \%$ \\
\hline External fixation & No & 23 & $53.5 \%$ \\
& Yes & 20 & $46.5 \%$ \\
\hline
\end{tabular}

\section{Discussion}

We did not find any significant risk factors for the occurrence of skin complications in patients undergoing surgical treatment of PMFs via the posterolateral approach. Time between trauma and definitive surgery, presence of fracture-dislocation, and use of external fixation before definitive repair were not significantly associated with either presence or absence of such complications. However, when we compared the rate of complications up to 2016 versus in 2017-2018, we observed a major reduction in the latter period, i.e., a higher risk
Table 2. Descriptive analysis of quantitative variables

\begin{tabular}{lccccc}
\hline Variable & N & Mean & SD & Minimum & Maximum \\
$\begin{array}{l}\text { Days elapsed from trauma } \\
\text { to surgery }\end{array}$ & 43 & 7.6 & 6.2 & 0 & 30 \\
$\begin{array}{l}\text { Weeks elapsed from } \\
\text { surgery to healing }\end{array}$ & 43 & 8.3 & 4.4 & 3 & 17 \\
\hline
\end{tabular}

of delayed healing in patients who underwent surgery at the beginning of the surgeons' learning curve.

The most prevalent complications of the posterolateral approach are related to the surgical wound, occurring in approximately $11 \%$ of cases $^{(20)}$. A systematic review of 19 articles including a total of 768 patients found that 39 (5\%) had skin complications ${ }^{(21)}$. In a large study, Little et al. ${ }^{(22)}$ evaluated the postoperative outcomes of 112 patients who underwent ORIF of ankle fractures via the posterolateral approach. Skin infection occurred in 5 patients. Another study evaluated 51 patients with PMF operated via the posterolateral approach, and found that 4 (7.8\%) developed skin complications in the postoperative period ${ }^{(20)}$. Conversely, another similar study found a twofold-higher incidence of skin complications (15.5\%)(12). In our study, the overall incidence of skin complications was 
Table 3. Comparison of explanatory variables between individuals with and without skin lesions

\begin{tabular}{|c|c|c|c|c|c|c|c|c|}
\hline & & \multicolumn{4}{|c|}{ Skin lesion } & \multirow{3}{*}{ OR } & \multirow{3}{*}{$95 \%$ Cl } & \multirow{3}{*}{ P-value } \\
\hline & & \multicolumn{2}{|c|}{ No } & \multicolumn{2}{|c|}{ Yes } & & & \\
\hline & & N & $\%$ & N & $\%$ & & & \\
\hline \multirow[t]{2}{*}{ Fracture-dislocation } & No & 13 & $56.5 \%$ & 10 & $43.5 \%$ & 1.00 & - & - \\
\hline & Yes & 11 & $55.0 \%$ & 9 & $45.0 \%$ & 1.06 & {$[0.32 ; 3.55]$} & 0.920 \\
\hline \multirow[t]{2}{*}{ External fixation } & No & 16 & $51.6 \%$ & 15 & $48.4 \%$ & 1.00 & - & - \\
\hline & Yes & 8 & $66.7 \%$ & 4 & $33.3 \%$ & 0.53 & {$[0.13 ; 2.14]$} & 0.376 \\
\hline Days elapsed from trauma to surgery & Mean & \multicolumn{2}{|c|}{7.8} & \multicolumn{2}{|c|}{7.3} & 0.98 & {$[0.89 ; 1.09]$} & 0.766 \\
\hline
\end{tabular}

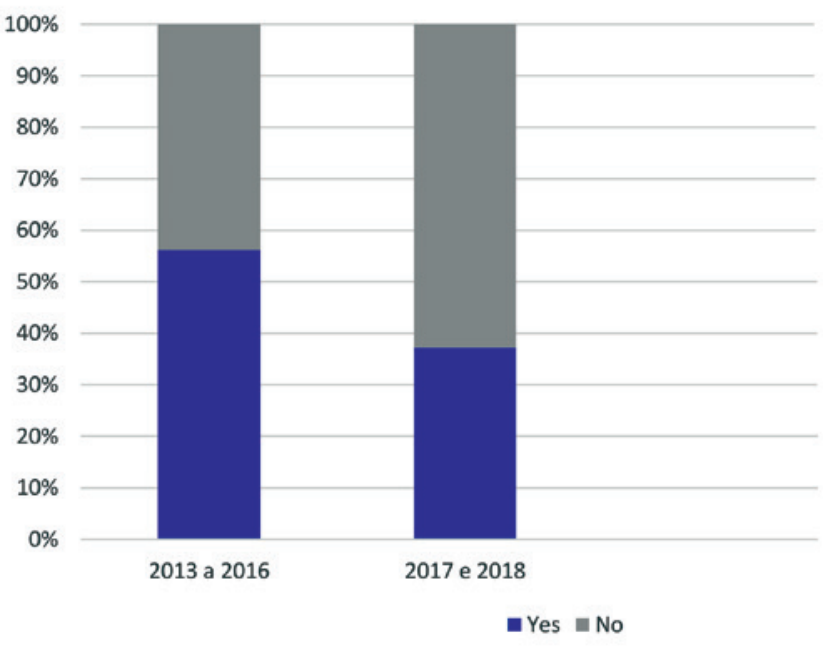

Figure 2. Patients who experienced skin complications, stratified by years.

44.2\%. One of the reasons for this discrepancy between our data and the literature is the fact that surgeons continue to adapt to the unique features of this approach. Furthermore, the cutoff used to define delayed healing was not reported in any of the articles found in the literature. This may bias analysis of the time point at which delayed healing can be considered an early complication.

Abdelgawad et al. ${ }^{(23)}$ argue that surgical wound dehiscences after the posterolateral approach are less disastrous than those occurring with the anterior, lateral and medial approaches, as the hardware is placed deeper and there is greater soft-tissue coverage. The results of the present study corro- borate this hypothesis, as no patient needed major intervention to address skin complications.

Unlike in the aforementioned study, however, we did not find any publications in the literature that considered the rate of skin complications to be a relevant factor in defining the optimal surgical approach to ankle fractures. We consider the PLA to be an excellent approach in addressing more complex fractures, but it has a steep learning curve. This is confirmed by the progressive decrease in incidence of complications over the years in the present study. Our decision to include patients who underwent surgery at three tertiary hospitals further highlights the importance of caution and expertise when performing ORIF via the posterolateral route.

The present study has some limitations. The retrospective design prevented further analysis of risk factors for the development of complications. The sample was small, consisting of only 43 individuals. Thus, the study was underpowered by our own calculation, which required a minimum of 134 patients divided into two groups to enable reliable comparison. Further studies with adequate sample sizes, a prospective design, and a clear definition of the main risk factors are needed to understand which factors have a negative impact on early complications of the posterolateral approach.

\section{Conclusion}

Patients who undergo open reduction and internal fixation of posterior malleolus fractures via the posterolateral route at the beginning of the learning curve are a greater risk of developing skin complications, demonstrating the importance of this approach being restricted to more experienced surgeons. However, these complications had no impact on the need for revision surgery. The posterolateral approach thus remains an important route in the treatment of complex ankle fractures. 
Authors' contributions: Each author contributed individually and significantly to the development of this article: CMPT *( https://orcid.org/OOOO-00022503-8721) conceived and planned the activities that led to the study, wrote the article, approved the final version; RSC *(https://orcid.org/OOOO-00015388-475X) conceived and planned the activities that led to the study, performed the surgeries, participated in the review process, approved the final version; FASL *(https://orcid.org/0000-0001-5214-2420) conceived and planned the activities that led to the study, performed the surgeries, participated in the review process, approved the final version; DSB *(https://orcid.org/0000-0001-5404-2132) conceived and planned the activities that led to the study, performed the surgeries, participated in the review process, approved the final version; TAAS *(https://orcid.org/0000-0003-2333-2334) conceived and planned the activities that led to the study, performed the surgeries, participated in the review process, approved the final version; RZAP *(https:// orcid.org/0000-0001-9692-5283) conceived and planned the activities that led to the study, performed the surgeries, participated in the review process, approved the final version. ${ }^{*}$ ORCID (Open Researcher and Contributor ID) (iD).

\section{References}

1. Zhong S, Shen L, Zhao JG. Comparison of posteromedial versus posterolateral approach for posterior malleolus fixation in trimalleolar ankle fractures. Orthop Surg. 2017;9(1):69-76.

2. Tenenbaum S, Shazar N, Bruck N, Bariteau J. Posterior malleolus fractures. Orthop Clin North Am. 2017;48(1):81-9.

3. Kukkonen J, Heikkilä JT, Kyyrönen T, Mattila K, Gullichsen E. Posterior malleolar fracture is often associated with spiral tibial diaphyseal fracture: a retrospective study. J Trauma. 2006;60(5): 1058-60.

4. Koval KJ, Lurie J, Zhou W. Ankle fractures in the elderly: what you get depends on where you live and who you see. J Orthop Trauma. 2005;19(9):635-9.

5. Bartoníček J, Rammelt S, Tuček M, Naňka O. Posterior malleolar fractures of the ankle. Eur J Trauma Emerg Surg. 2015;41(6):587-600.

6. Tejwani NC, Pahk B, Egol KA. Effect of posterior malleolus fracture on outcome after unstable ankle fracture. J Trauma. 2010;69(3):666-9.

7. Nelson MC, Jensen NK. The treatment of trimalleolar fractures of the ankle. Surg Gynecol Obstet. 1940;71:509-14.

8. Lee HJ, Kang KS, Kang SY, Lee JS. Percutaneous reduction technique using a Kirschner wire for displaced posterior malleolar fractures. Foot Ankle Int. 2009; 30:157-9

9. Masuda V, Pereira V, Baumfeld D, Neves C, Nery C, Mansur, NS. Fixation of posterior malleolus fractures through posterior access in the ankle. J Foot Ankle. 2018;12(3):193-8.

10. Veltman ES, Halma JJ, Gast A. Longterm outcome of 886 posterior malleolar fractures: a systematic review of the literature. Foot Ankle Surg. 2016;22(2),73-7.

11. Nagelkerke NJ. A note on a general definition of the coefficient of determination. Biometrika. 1991;78(3);691-2.

12. Hoogendoorn JM. Posterior malleolar open reduction and internal fixation through a posterolateral approach for trimalleolar fractures. JBJS Essent Surg Tech. 2017;7(4):e31.
13. Carmont MR, Davies MB. Buttress plate stabilization of posterior malleolar ankle fractures: a familiar technique through an unfamiliar approach. Foot Ankle. 2008;22(5):359-64.

14. Kadakia AR, Dekker 2nd RG, Ho BS. Acute Achilles tendon ruptures: an update on treatment. J Am Acad Orthop Surg. 2017;25(1):23-31.

15. Agresti A. Categorical analysis. New York: John Wiley; 2002

16. Efroymson MA. Multiple regression analysis. In: Mathematical methods for digital computers. New York: John Wiley; 1960. p. 191-203.

17. Hosmer DW, Lemeshow S. Applied logistic regression. New York: Wiley; 2000.

18. Minihane KP, Lee C, Ahn C, Zhang LQ, Merk BR. Comparison of lateral locking plate and antiglide plate for fixation of distal fibular fractures in osteoporotic bone: a biomechanical study. J Orthop Trauma. 2006; 20:562-6.

19. Forberger J, Sabandal PV, Dietrich M, Gralla J, Lattmann T, Platz A. Posterolateral approach to the displaced posterior malleolus: functional outcome and local morbidity. Foot Ankle Int. 2009; 30(4):309-14.

20. Amorosa LF, Brown GD, Greisberg J. A surgical approach to posterior pilon fractures. J Orthop Trauma. 2010;24:188-93.

21. Webb J, Moorjani N, Radford M. Anatomy of the sural nerve and its relation to the Achilles tendon. Foot Ankle Int. 2000; 21(6):475-7

22. Little MT, Berkes MB, Lazaro LE, Sculco PK, Helfet DL, Lorich DG. Complications following treatment of supination external rotation ankle fractures through the posterolateral approach. Foot Ankle Int. 2013;34(4):523-9

23. Abdelgawad AA, Kadous A, Kanlic E. Posterolateral approach for treatment of posterior malleolus fracture of the ankle. J Foot Ankle Surg. 2011;50(5):607-11. 